\title{
Electronic coherence within the semiclassical field-induced surface hopping method: Strong field quantum control in $\mathbf{K}_{2}$
}

\author{
Jens Petersen ${ }^{a}$ and Roland Mitrićc ${ }^{* a}$ \\ Received Xth XXXXXXXXXX 20XX, Accepted Xth XXXXXXXXX 20XX \\ First published on the web Xth $X X X X X X X X X X 200 X$ \\ DOI: 10.1039/b000000x
}

We demonstrate that the semiclassical field-induced surface hopping (FISH) method (Mitrić et al., Phys. Rev. A 2009, 79, 053416) accurately describes the selective coherent control of electronic state populations. On the example of the strong field control in the potassium dimer using phase-coherent double pulse sequences, we present a detailed comparison between FISH simulations and exact quantum dynamics. We show that for short pulses the variation of the time delay between the subpulses allows for a selective population of the desired final state with high efficiency. Furthermore, also for pulses of longer time duration, when substantial nuclear motion takes place during the action of the pulse, optimized pulse shapes can be obtained which lead to selective population transfer. For both types of pulses, the FISH method almost perfectly reproduces the exact quantum mechanical electronic population dynamics, fully taking account of the electronic coherence, and describes the leading features of the nuclear dynamics accurately. Due to the significantly higher computational efficiency of FISH as a trajectorybased method compared to full quantum dynamics simulations, this offers the possibility to theoretically investigate control experiments on realistic systems including all nuclear degrees of freedom.

\section{Introduction}

Light-induced ultrafast dynamical processes in molecular systems are governed by transitions between the electronic states, which arise either through the interaction with electromagnetic fields or due to the intrinsic nonadiabatic coupling. The theoretical description of such phenomena which are fundamental for photochemistry requires efficient methods for performing coupled electron-nuclear dynamics simulations. The largest obstacles preventing the use of fully quantum mechanical approaches for complex systems are the inability to precalculate accurate global potential energy surfaces in multidimensional systems as well as the tremendous cost of full quantum wavepacket dynamics simulations which restricts their application to systems containing only few atoms. Thus, the direct dynamics employing classical trajectories propagated "on the fly" using various quantum chemical methods has become one of the major computational tools for the treatment of complex systems. In the field of nonadiabatic dynamics, a particularly successful method is the mixed quantum-classical dynamics based on Tully's surface hopping approach ${ }^{1,2}$ which has in recent years been combined with ab initio molecular dynamics and has provided valuable insight into the mechanisms of various photochemical and photophysical processes ${ }^{3-22}$. It should be noted that while most nonadiabatic dynamics simulations have been carried out without explicitly considering

a Freie Universität Berlin, Fachbereich Physik, Arnimallee 14, D-14195 Berlin, Germany; E-mail: mitric@zedat.fu-berlin.de the electronic excitations induced by light, there are several important application areas such as e.g. ultrafast spectroscopy or coherent control which require the inclusion of laser fields into the dynamics simulations. We have recently developed the field-induced surface hopping method (FISH) ${ }^{23}$ which allowed us to simulate the laser-driven multistate dynamics in complex molecular systems. Our approach is based on the idea to combine classical nuclear dynamics with quantum mechanical electronic state population dynamics and to allow the trajectories to switch between the electronic states in analogy with Tully's surface hopping method. However, the switching probability is determined both by nonadiabatic couplings as well as by external electric fields. This method is widely applicable for the theoretical description of light-induced dynamical processes in molecular systems. Specifically, we have recently simulated spectroscopic observables such as timeresolved photoelectron spectra ${ }^{24-26}$ or harmonic emission ${ }^{27}$.

A particularly important application area of FISH simulations is the coherent control of molecular processes by optimally shaped laser fields. Stimulated by theorical concepts $^{28-32}$, the progress in laser pulse shaping technology has led to numerous experimental realizations allowing for manipulation of photochemical and photophysical processes on the time scale of nuclear ${ }^{33-37}$ and electronic motion ${ }^{38-41}$ and for control of the outcome and yield of chemical reactions ${ }^{42-44}$. The most widely used control strategy relies on the iterative optimization of the control field in the closed-loop learning scheme utilizing the response of the molecular system as a 
feedback signal, as theoretically proposed by Judson and Rabitz $^{45}$ and subsequently applied in several pioneering experimental studies ${ }^{46-50}$. However, in this way complex pulse forms are usually obtained and their interpretation is often far from being obvious. Therefore, the efficient simulation of coherent dynamics driven by shaped laser fields is highly desirable in order to reveal the mechanism underlying the control and to establish the connection between the experimentally optimized pulse shapes and the intrinsic dynamical processes. In this context, as already demonstrated on several examples, our FISH method offers a uniquely powerful tool for the simulation of control experiments in complex molecular systems. Since the electric field is directly included in the dynamics simulations, it can be theoretically optimized in analogy with the experimental closed-loop learning approach, which we have illustrated on the example of selective isomerization of a Schiff base molecular switch ${ }^{23}$. Furthermore, the FISH method can also directly utilize experimentally optimized laser fields in order to reveal the control mechanism, as we have shown on the example of optimal dynamic discrimination of two spectroscopically almost identical flavin molecules ${ }^{51}$. The applicability of the FISH method to the control of dynamics in the condensed phase has also been recently introduced in the framework of the quantum mechanical/molecular mechanical (QM/MM) approach ${ }^{52}$.

Since the FISH method employs classical trajectories for the nuclear dynamics, the fundamental question has to be addressed to which extent it can take into account the electronic and nuclear coherence effects which are at the heart of the coherently controlled dynamics. In our previous work we have already demonstrated that the FISH method can perfectly reproduce the coherent Rabi oscillations between two electronic states in a two-level model system. Moreover, we have shown on several one-dimensional systems that the FISH method almost perfectly reproduces the results of full quantum dynamics simulations ${ }^{23,27}$. In this contribution, we focus on the strong field control and investigate systematically the ability of our FISH method to describe coherent processes in a system with several coupled electronic states. We have chosen the potassium dimer as a prototype since accurate potential energy curves and transition dipole moments are available. Moreover, the strong field control using the selective population of dressed states (SPODS) scheme, which was previously experimentally applied to potassium atoms ${ }^{53-55}$, has been already demonstrated on this molecular example using quantum dynamics simulations ${ }^{56}$. Within SPODS, the excitation to a manifold of excited electronic states by a phase coherent double pulse sequence is used to manipulate the final state population. Almost perfect selectivity in populating different target states has been achieved by varying the time delay between the two subpulses. Therefore, due to its conceptual clarity, the SPODS control scheme is particularly suitable for system- atic investigation of coherent control in the framework of the semiclassical FISH method.

We demonstrate that the FISH method accurately describes the coherent electronic processes induced within the SPODS control scheme and gives results which are in almost perfect agreement with the exact quantum dynamics simulations. Thus, due to its applicability to complex systems with many degrees of freedom, the FISH method is suitable for the simulation of coherent control in systems ranging from polyatomic (bio)molecules and clusters to complex nanostructures and supramolecular assemblies or systems interacting with their environment such as solvent or surfaces.

The paper is organized as follows: First, the field-induced surface hopping method is briefly reviewed and the computational details are presented in Section 2. Subsequently, in Section 3 the results are presented and discussed. Finally, conclusions are given in Section 4

\section{Theoretical Formulation}

\subsection{Field-induced surface hopping (FISH)}

The detailed description of FISH simulations ${ }^{23}$ as well as several applications to complex molecular systems ${ }^{23-27,51,52}$ have been provided previously. Briefly, the idea of the FISH method is to extend the applicability of surface hopping simulations to laser-driven dynamics by including the coupling between electronic states due to the interaction with the electromagnetic field. In this way, classical trajectories are propagated in a manifold of several electronic states, and the population transfer is described by allowing the trajectories to switch between the states according to quantum mechanically calculated transition probabilities. For performing the FISH simulations, three steps are needed: First, the initial ensemble of trajectories is generated, e.g. by sampling the quantum mechanical Wigner distribution function. Second, each trajectory is propagated in the manifold of ground and excited electronic states using classical molecular dynamics. Notice, that so far the FISH simulations have been performed both in the framework of ab initio methods (TDDFT) as well as using semiempirical methods for the electronic structure. Third, parallel to the propagation of classical trajectories, along each trajectory the time-dependent Schrödinger equation is solved in the manifold of adiabatic electronic states coupled by the laser field $\vec{E}(t)$ and by the nonadiabatic coupling terms $D_{i j}=\left\langle\psi_{i} \mid \frac{d \psi_{j}}{d t}\right\rangle$

$$
\begin{aligned}
i \hbar \dot{c}_{i}(t)= & E_{i}(\mathbf{R}(t)) c_{i}(t) \\
& -\sum_{j}\left[i \hbar D_{i j}(\mathbf{R}(t))+\vec{\mu}_{i j}(\mathbf{R}(t)) \cdot \vec{E}(t)\right] c_{j}(t),
\end{aligned}
$$

From the quantum mechanical amplitudes $c_{i}(t)$ the density matrix elements are calculated as $\rho_{i j}=c_{i}^{*} c_{j}$. Since the di- 
agonal elements of the density matrix correspond to the populations of the respective electronic states, their change can be used to devise a stochastic procedure for switching the trajectories between different electronic states. In this way, the hopping probabilities are determined in each nuclear time step from the change of the quantum electronic state populations $\rho_{i i}$ according to

$$
P_{i \rightarrow j}=\Theta\left(-\dot{\rho}_{i i}\right) \Theta\left(\dot{\rho}_{j j}\right) \frac{-\dot{\rho}_{i i}}{\rho_{i i}} \frac{\dot{\rho}_{j j}}{\sum_{k} \Theta\left(\dot{\rho}_{k k}\right) \dot{\rho}_{k k}} \Delta t
$$

and used in a stochastic process to decide if a state switch occurs ${ }^{27,57}$. The $\Theta$ functions have a value of one for positive arguments and of zero otherwise. The smooth electronic state population as a function of time is then obtained by averaging over the ensemble of trajectories. Notice, that FISH simulations can be straightforwardly extended in order to include other types of couplings such as e.g. magnetic dipole coupling which can be used to simulate the dynamics of chiral systems driven by polarized laser fields. In the same spirit, the FISH method has been recently also extended in order to include the spin-orbit coupling ${ }^{58}$. Thus, the FISH method is a general framework which allows for the simulation and control of laser-driven processes in complex molecular systems. We wish to emphasize that in our simulations, we have specifically considered nuclear dynamics on field-free potential energy surfaces and have not explicitly accounted for the deformation of the potentials due to the laser fields. However, since the deformed light-induced potentials can be regarded as linear combinations of the unperturbed potentials which are recovered after the pulses have ceased, it makes in principle no difference which potentials are used for the propagation. In particular, the effects of resonant Stark shift which are the fundament of the SPODS scheme are fully covered also when the nuclei are propagated on the field-free potentials. The sole contribution neglected in this approach is the additional force component originating from dipole moment derivatives, which plays a significant role only at very high field strengths. Although there have recently been several attempts to straightforwardly extend our FISH method by including these effects, the applicability of such approaches has so far been restricted only to one-dimensional model systems ${ }^{58,59}$. Its extension towards real systems would require the analytic "on the fly" calculation of dipole moment derivatives in electronically excited state which would severly increase the computational cost of the simulations.

\subsection{Computational Procedures}

2.2.1 Ab initio potential energy curves and transition dipole moments. Both the quantum dynamical as well as the FISH simulations have been performed using precalculated potential energy curves and distance-dependent electric tran-
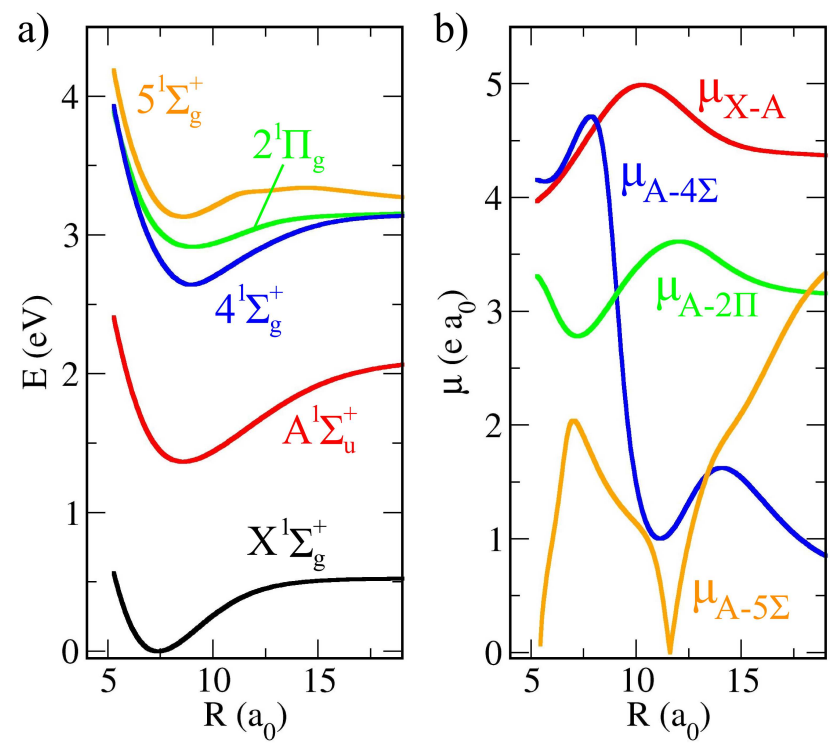

Fig. 1 a) CAS-MRCI potential energy curves for the states $X^{1} \Sigma_{g}^{+}$, $A^{1} \Sigma_{u}^{+}, 4^{1} \Sigma_{g}^{+}, 2^{1} \Pi_{g}^{+}$and $5^{1} \Sigma_{g}^{+}$of K2. b) Transition dipole moments between the $A^{1} \Sigma_{u}^{+}$and the $X^{1} \Sigma_{g}^{+}, 4^{1} \Sigma_{g}^{+}, 2^{1} \Pi_{g}^{+}$and $5^{1} \Sigma_{g}^{+}$states as a function of the internuclear distance.

sition dipole moments for the $\mathrm{K}_{2}$ dimer. For this purpose, the high level multireference configuration interaction (MRCI) method $^{60-62}$ was employed as implemented in the MOLPRO program package ${ }^{63}$. As reference states in the MRCI calculation, the state-averaged complete active space self-consistent field (CAS-SCF) wavefunctions ${ }^{64,65}$ were used. The $\mathrm{K}$ atoms were described using a 1-electron effective core potential ${ }^{66}$ together with a $(7 \mathrm{~s} 5 \mathrm{p} 7 \mathrm{~d} 2 \mathrm{f}) /[6 \mathrm{~s} 5 \mathrm{p} 5 \mathrm{~d} 2 \mathrm{f}]$ Gaussian basis set ${ }^{67}$ as well as the core polarization potential from Ref. ${ }^{68}$. The active space for the CAS-SCF calculations was constructed from the one occupied and 15 virtual orbitals. Subsequently, the MRCI calculations including single and double excitations from all reference wavefunctions have been performed. This approach has previously been proven to yield precise results for $\mathrm{K}_{2} 67,69$. The potential energy curves for the $X^{1} \Sigma_{g}^{+}, A^{1} \Sigma_{u}^{+}, 4^{1} \Sigma_{g}^{+}, 2^{1} \Pi_{g}$ and $5^{1} \Sigma_{g}^{+}$states as well as the transition dipole moments between the $X^{1} \Sigma_{g}^{+}$and $A^{1} \Sigma_{u}^{+}$as well as between the $A^{1} \Sigma_{u}^{+}$and the higher-lying $4^{1} \Sigma_{g}^{+}, 2^{1} \Pi_{g}^{+}$and $5^{1} \Sigma_{g}^{+}$states have been calculated in the range between 5.25 and $25.0 a_{0}$.

2.2.2 Quantum dynamics simulations. The quantum dynamics calculations have been carried out employing the grid-based numerical solution of the nuclear Schrödinger equation using a second-order difference propagator ${ }^{70}$. The values of the potential energy as well as of the transition dipole moments at the 256 grid points for nuclear distances between 4 and $15 a_{0}$ were obtained by B-spline interpolation. As initial 


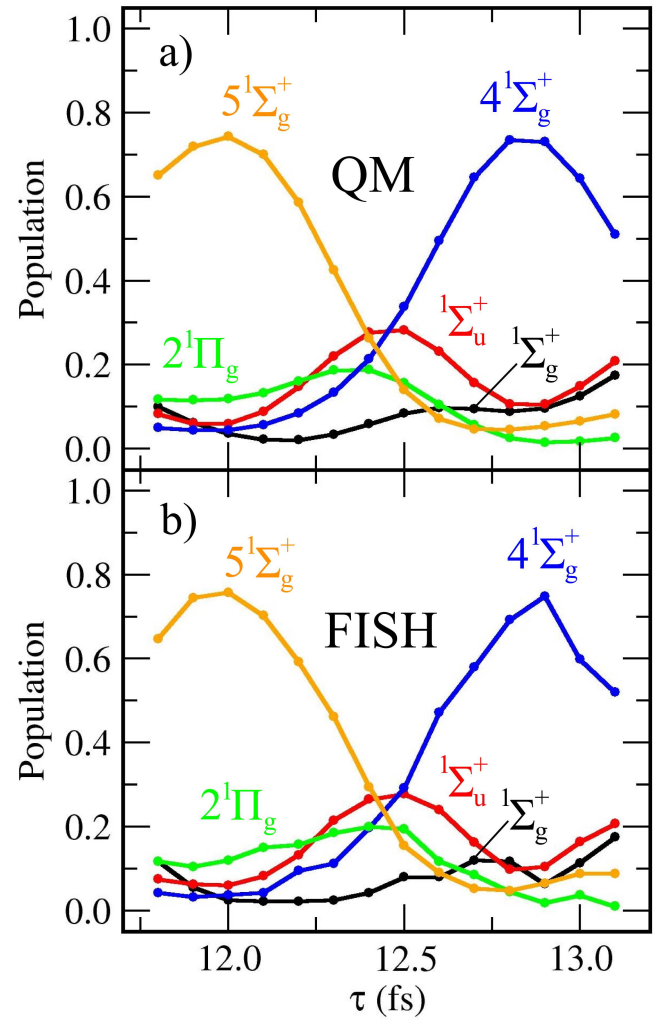

Fig. 2 Final state population after excitation with a short phase-coherent double pulse laser field as a function of the time delay $\tau$ between the two subpulses [cf. Eq. (3)].

condition, the lowest vibrational eigenstate of the electronic ground state $X^{1} \Sigma_{g}^{+}$was chosen. The time step for the dynamics was $0.005 \mathrm{fs}$, and the propagation was performed over 60 fs. The laser coupling of the electronic states was described by the electric dipole interaction $-\vec{\mu}_{i j}(\mathbf{R}(t)) \cdot \vec{E}(t)$. The electric field was parameterized in the time domain according to

$$
E(t)=\left(E_{1} e^{-2 \ln 2(t / \Delta t)^{2}}+E_{2} e^{-2 \ln 2((t-\tau) / \Delta t)^{2}} e^{i \omega_{0} \tau}\right) e^{i \omega_{0} t}
$$

with a frequency $\omega_{0}$ of $1.49 \mathrm{eV}(830 \mathrm{~nm})$, a width of $\Delta t=$ $14.1 \mathrm{fs}$ and amplitudes for the subpulses of $E_{1}=0.0011$ a.u. $\left(4.17 \cdot 10^{10} \mathrm{~W} / \mathrm{cm}^{2}\right)$ and $E_{2}=0.005$ a.u. $\left(8.78 \cdot 10^{11} \mathrm{~W} / \mathrm{cm}^{2}\right)$. The polarization of the field was assumed to be $45^{\circ}$ with respect to the internuclear axis of the molecule. For the pulse delay $\tau$, different values in the range from 11.8 to $13.1 \mathrm{fs}$ have been employed. Experimentally, such ultrashort femtosecond pulse pairs with precisely adjusted time delay (up to a precision of 0.3 attoseconds) have been recently realized by Baumert et al. and have been applied to the SPODS control in potassium atoms ${ }^{71}$.
2.2.3 FISH simulations. For the FISH dynamics, 300 initial conditions were sampled from a canonical Wigner distribution function at $10 \mathrm{~K}$, and the nuclei were propagated classically in the respective electronic states by solving the Newtonian equations of motion using the velocity Verlet algorithm ${ }^{72,73}$ with a time step of 0.2 fs. The total propagation time was the same as for the quantum dynamics. The necessary forces have been obtained "on the fly" from the numerical gradients of the potential energy curves. According to the FISH procedure, the trajectories were allowed to switch between the electronic states in a probabilistic manner. For this purpose, the time-dependent Schrödinger equation (1) was integrated numerically in the basis of the electronic states along the nuclear trajectories, employing a time step of $8 \cdot 10^{-5}$ fs. Notice, that in the present contribution only the dipole coupling between the electronic states was taken into account and the nonadiabatic couplings present in Eq. (1) were neglected. The probabilities for hoppings between the electronic states were calculated from the quantum mechanical state populations $\rho_{i i}=c_{i}^{*} c_{i}$ according to Eq. (2). In order to improve the statistics of the hopping events, the whole ensemble of initial conditions was propagated twice.

2.2.4 Pulse optimization. The ability of longer laser pulse sequences to selectively populate a chosen excited state has been examined by optimizing a double pulse sequence in a restricted parameter space. For this purpose, the field parameterization given in Eq. (3) has been generalized to

$$
\begin{aligned}
E(t)= & E_{1} e^{-2 \ln 2\left(\left(t-\tau_{1}\right) / \Delta t_{1}\right)^{2}} e^{i \omega_{1}\left(t-\tau_{1}\right)} \\
& +E_{2} e^{-2 \ln 2\left(\left(t-\tau_{2}\right) / \Delta t_{2}\right)^{2}} e^{i \omega_{2}\left(t-2 \tau_{1}+\tau_{2}\right)} .
\end{aligned}
$$

Using a genetic algorithm ${ }^{74}$ the field parameters have been optimized with the target to maximize the population of the $2^{1} \Pi_{g}$ state after the field has ceased. The optimization procedure was performed employing FISH simulations with a smaller ensemble of 72 trajectories.

\section{Results and discussion}

The calculated potential energy curves for $\mathrm{K}_{2}$ which serve as a basis for quantum dynamical and FISH simulations are presented in Fig. 1a). We consider the electronic ground state $X^{1} \Sigma_{g}^{+}$and four electronically excited states $\left(A^{1} \Sigma_{u}^{+}, 4^{1} \Sigma_{g}^{+}\right.$, $2^{1} \Pi_{g}$ and $\left.5^{1} \Sigma_{g}^{+}\right)$. The first optically allowed excited state $\left(A^{1} \Sigma_{u}^{+}\right)$can be easily reached in a single photon process with $830 \mathrm{~nm}$ light. The three higher excited states $4{ }^{1} \Sigma_{g}^{+}, 2^{1} \Pi_{g}$ and $5^{1} \Sigma_{g}^{+}$lie closely together and are, also using $830 \mathrm{~nm}$ excitation, in principle accessible from the $A^{1} \Sigma_{u}^{+}$state. This opens a possiblity to control the population of these higher excited states by using phase-coherent double pulses as previously demonstrated by Wollenhaupt and Baumert ${ }^{56}$. The rele- 


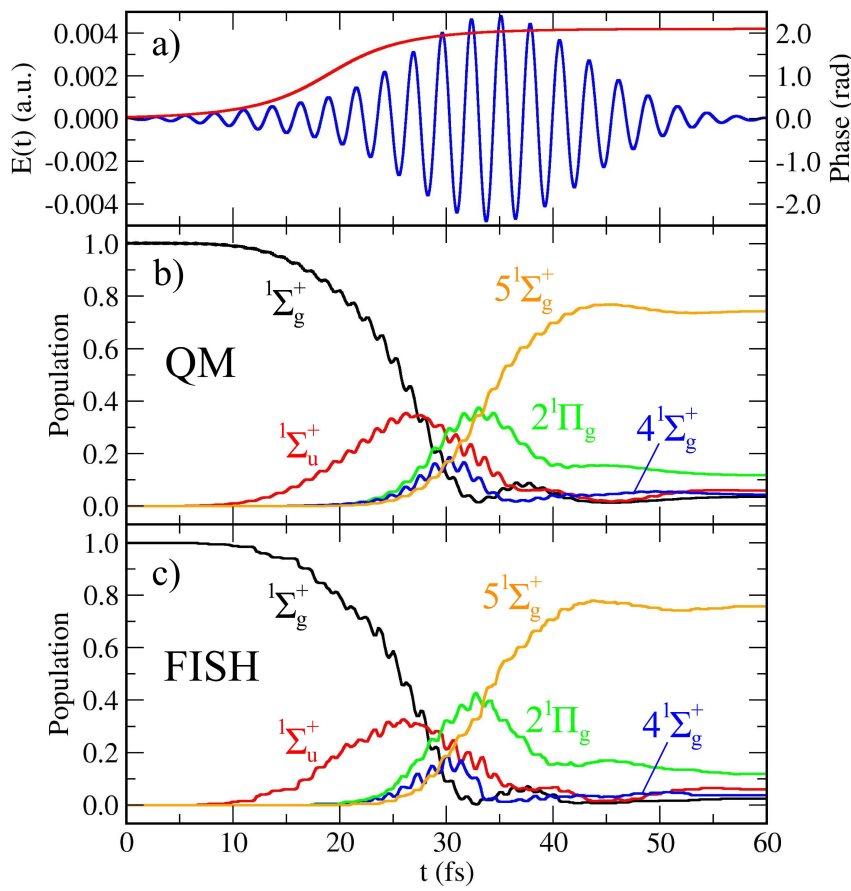

Fig. 3 (a) Temporal field strength (blue) and relative temporal phase (red) of the double pulse with time delay $\tau=12.0 \mathrm{fs}$. The field strength is given in atomic units $\left.\left.\left(1 a . u .=5.14 \cdot 10^{11} \mathrm{~V} / \mathrm{m}\right) . \mathrm{b}\right) / \mathrm{c}\right)$ Time-dependent electronic state populations obtained by quantum dynamics (b) and by employing the FISH method (c).

vant transition dipole moments between the considered states strongly depend on the internuclear distance as shown in Fig. $1 b)$.

In order to explore the ability of the FISH method to describe coherent control of the electronic state population we have, following Ref. ${ }^{56}$, first performed FISH simulations using excitation with the field given in Eq. (3) and systematically varying the parameter $\tau$ in the range from 11.8 fs to $13.1 \mathrm{fs}$. For comparison, numerically exact quantum dynamics simulations have been also performed. The resulting dependence of the final electronic state population on the time delay $\tau$ is presented in Fig. 2. As can be seen from the quantum dynamical simulations, the time delay has a strong influence on the final population of the $4^{1} \Sigma_{g}^{+}$and $5^{1} \Sigma_{g}^{+}$states such that almost complete reversal of the corresponding populations can be achieved. Specifically, maximal population of the $4^{1} \Sigma_{g}^{+}$ state of $\sim 75 \%$ is obtained for $\tau=12.0$ fs while the population of the $5^{1} \Sigma_{g}^{+}$state is kept below $10 \%$ in this case. In contrast, for $\tau=12.9$ fs the population of the $5^{1} \Sigma_{g}^{+}$is maximized while the population of the $4^{1} \Sigma_{g}^{+}$remains lower than $10 \%$. As can be seen from Fig. 2b), the semiclassical FISH simulations almost perfectly reproduce the quantum dynamics results of the final electronic state populations.

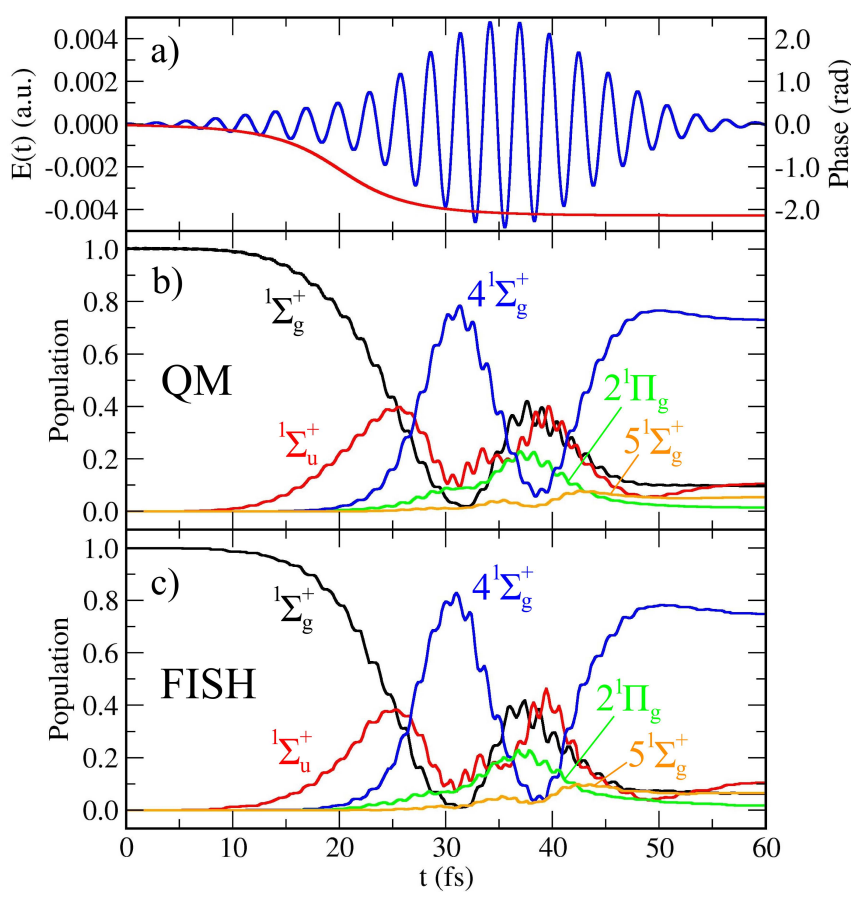

Fig. 4 (a) Temporal field strength (blue) and relative temporal phase (red) of the double pulse with time delay $\tau=12.9 \mathrm{fs}$. b)/c) Time-dependent electronic state populations obtained by quantum dynamics (b) and by employing the FISH method (c).

In order to examine the details of the electronic state population dynamics within the FISH method and to validate them against the full quantum mechanical results we present in Figs. 3 and 4 the resulting time-dependent populations obtained using the values of $\tau=12.0$ fs and 12.9 fs which correspond to the maximal final populations of the $4^{1} \Sigma_{g}^{+}$or $5^{1} \Sigma_{g}^{+}$states, respectively. In Fig. 3a the excitation field (blue line) together with the relative temporal phase (red line) is shown. The phase smoothly varies between zero and $\sim 2.1$. The population dynamics obtained fully quantum mechanically and in the framework of the FISH method are presented in Fig. 3b) and 3c). In both cases, the ground state population is transferred via transient occupation of the $A^{1} \Sigma_{u}^{+}$and $2^{1} \Pi_{g}$ finally to the $5^{1} \Sigma_{g}^{+}$ state. The final population of this state reaches in both cases about $75 \%$. Overall, the time-dependent populations obtained using the FISH method follow closely the full quantum mechanical results. Notice, that our quantum mechanical and FISH simulations do not employ the rotating wave approximation, in contrast to the simulations of Ref. ${ }^{56}$. Therefore, our electronic state populations exhibit additional small oscillations corresponding to the counter-rotating corrections to the rotating wave approximation.

For $\tau=12.9$ fs the overall envelope of the electric field is unchanged, but the relative temporal phase varies now from zero 
to $\sim-2.1$ as shown in Fig. 4a). This phase change leads to dramatically different dynamics. The resulting state populations are presented in Fig. 4b for the full quantum and in Fig. $4 \mathrm{c}$ for the FISH simulation. In both cases, after initial population of the $A^{1} \Sigma_{u}^{+}$state, the $4^{1} \Sigma_{g}^{+}$state starts to be populated immediately and reaches a maximal value of $80 \%$ after 30 fs. Subsequently, large-amplitude Rabi oscillations lead to population transfer back to the $X^{1} \Sigma_{g}^{+}$and $A^{1} \Sigma_{u}^{+}$states, and also partly to the $2^{1} \Pi_{g}$ state. After 38 fs the population of $4{ }^{1} \Sigma_{g}^{+}$ state has diminished to a value of only $4 \%$. However, after 40 fs the $4^{1} \Sigma_{g}^{+}$state begins again to be populated, reaching a final value of almost $75 \%$ after the pulse has ceased. Again, the comparison between full quantum and FISH dynamics shows perfect agreement. These findings clearly indicate that the FISH method is adequate for simulation and control of coherent electronic state population dynamics in a manifold of several coupled electronic states. Since the FISH method, as we have previously demonstrated, can be straightforwardly coupled with either ab initio or semiempirical molecular dynamics, this opens a possibility to control coherent electronic processes as well as to simulate time-resolved spectroscopies in complex systems such as biomolecules interacting with the environment, which is far beyond the reach of full quantum dynamics simulations.

The ability of the FISH method to correctly describe the coupled electron-nuclear dynamics in a manifold of several electronic states is not restricted to the short pulse limit, in which no significant nuclear motion occurs, but also applies to the case in which the laser pulse duration is longer and the internuclear separation changes considerably. In order to illustrate this, we have optimized a double pulse sequence of longer time duration with the aim to maximize the final population of the $2^{1} \Pi_{g}$ state. The resulting pulse as well as the time-dependent electronic state populations for both the quantum dynamical and the FISH simulation are shown in Fig. 5, while the optimized pulse parameters are given in Table 1 . The first subpulse induces population transfer between the ground state and the excited ${ }^{1} \Sigma_{u}^{+}, 5^{1} \Sigma_{g}^{+}$and $2^{1} \Pi_{g}$ states until $\mathrm{t} \approx 130$ fs. Under the influence of the second pulse at later times only Rabi cycles between the ground state and the $2^{1} \Pi_{g}$ state are induced, while the populations of all other states remain very small. Finally, when the field ceases, a $2^{1} \Pi_{g}$ population of $\sim 70 \%$ is reached. Comparison between the exact quantum dynamics (Fig. 5b) and FISH (Fig. 5c) clearly shows that also in the case where the the wavepacket moves substantially during the action of the pulse, the FISH electronic state populations agree very well with the full quantum dynamics results. The time evolution of the probability distributions for the internuclear distance $R$ and the corresponding momentum $P$ is illustrated in Fig. 6 for both the full quantum dynamics and the FISH dynamics employing the optimized pulse from Fig. 5. As can be seen from Fig. 6a) the main part of the

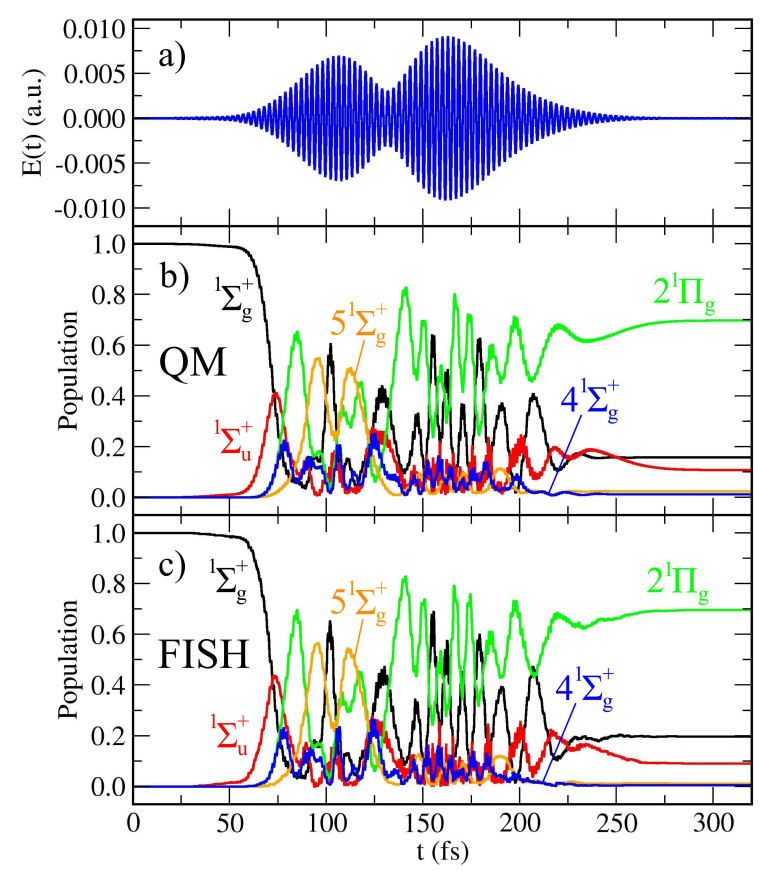

Fig. 5 (a) Temporal field strength of the optimized long double pulse with time duration of about $150 \mathrm{fs}$. b)/c) Time-dependent electronic state populations obtained by quantum dynamics (b) and by employing the FISH method using the ensemble of 300 trajectories (c). 
Table 1 Optimized pulse parameters for maximizing the population of the $2^{1} \Pi_{g}$ state [cf. Eq. (4)].

\begin{tabular}{rr}
\hline Parameter & Value \\
\hline $\mathrm{E}_{1}$ & $4.713 \cdot 10^{-3}$ a.u. \\
$\omega_{1}$ & $1.521 \mathrm{eV}$ \\
$\mathrm{w}_{1}$ & $73.54 \mathrm{fs}$ \\
$\mathrm{t}_{0,1}$ & $120.8 \mathrm{fs}$ \\
$\mathrm{E}_{2}$ & $8.390 \cdot 10^{-3}$ a.u. \\
$\omega_{2}$ & $1.473 \mathrm{eV}$ \\
$\mathrm{w}_{2}$ & $86.48 \mathrm{fs}$ \\
$\mathrm{t}_{0,2}$ & $151.6 \mathrm{fs}$ \\
\hline
\end{tabular}

position wavepacket, starting from the ground state minimum around $7.4 a_{0}$, steadily moves to larger internuclear distances until it reaches the outer turning point at $10.1 a_{0}$ after $\sim 350$ fs. Since the field has already ceased after $250 \mathrm{fs}$, the subsequent dynamics is dominated by the wavepacket oscillation in the $2^{1} \Pi_{g}$ state with a period of $\sim 750 \mathrm{fs}$. Similarly, also the momentum oscillates with this period [cf. 6c)]. The same behaviour is seen for the FISH simulations [cf. Fig. 6b) and d)]. Notice, that for comparison with the full quantum mechanical simulation the FISH probability densities for $R$ and $P$ have been obtained by convoluting each trajectory with a Gaussian function of width $0.15 a_{0}$ in position and $2.5 a_{0}^{-1}$ in momentum space and averaging over the whole ensemble. Although the overall agreement is very good, after $700 \mathrm{fs}$ the quantum dynamics simulation exhibits characteristic interference features which are a consequence of nuclear coherence. Such phenomena fundamentally cannot be present in FISH simulations due to the purely classical propagation of the nuclear degrees of freedom. However, we emphasize that the effect of the electronic coherence is fully accounted for since the electronic degrees of freedom are propagated quantum mechanically according to Eq. (1). Further insight into the quantum and FISH dynamics can be obtained by analyzing the dynamical processes in terms of the phase space distribution. For this purpose, the quantum mechanical nuclear wavepackets $\chi_{i}(R)$ have been Wigner transformed ${ }^{75}$ as

$$
\begin{aligned}
W(R, P) & =\sum_{i} W_{i}(R, P) \\
& =\frac{1}{2 \pi \hbar} \sum_{i} \int d Y \chi_{i}^{*}\left(R-\frac{Y}{2}\right) \chi_{i}\left(R+\frac{Y}{2}\right) e^{\frac{i}{\hbar} P Y}
\end{aligned}
$$

where the index $i$ denotes the respective electronic state. As can be seen from Fig. 7 the main part of the quantum mechanical Wigner distribution exhibits an elliptical motion in the phase space as it is expected for a bound potential. Interestingly, after $300 \mathrm{fs}$ negative contributions appear in the distribution which can be attributed partly to portions of the wavepacket moving in other states than the mainly populated $2^{1} \Pi_{g}$, and partly to quantum mechanical nuclear interferences.
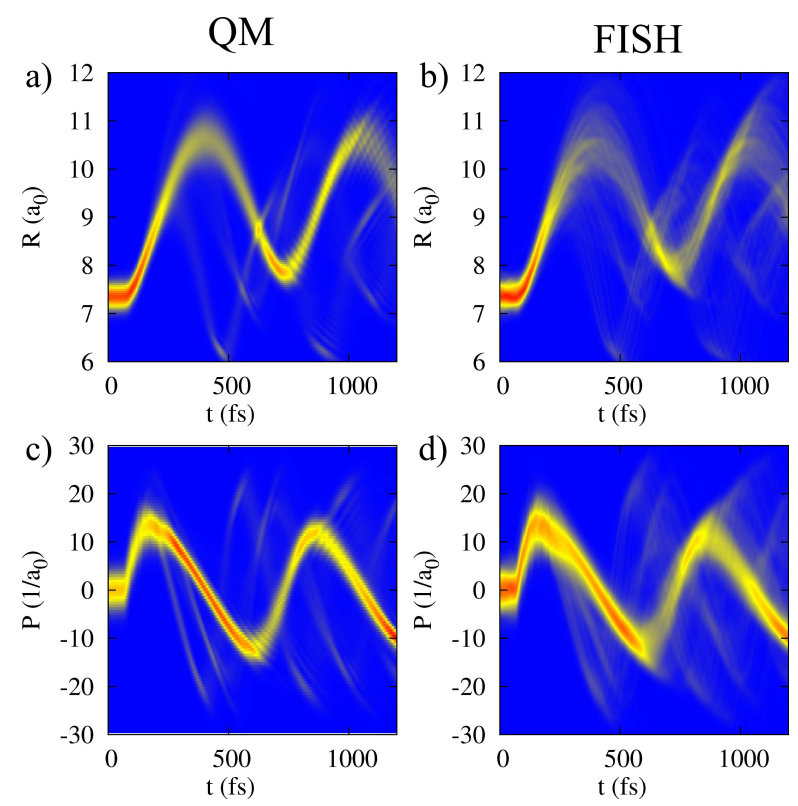

Fig. 6 Position and momentum probability distributions for the dynamics induced by the optimzed double pulse shown in Fig. 5a) employing quantum dynamics [a) and c)] as well as the FISH method [b) and d)]. The label $a_{0}$ denotes the Bohr radius, $a_{0}=0.529 \AA$. Blue color represents zero probability density, yellow and red denote increasing positive values.

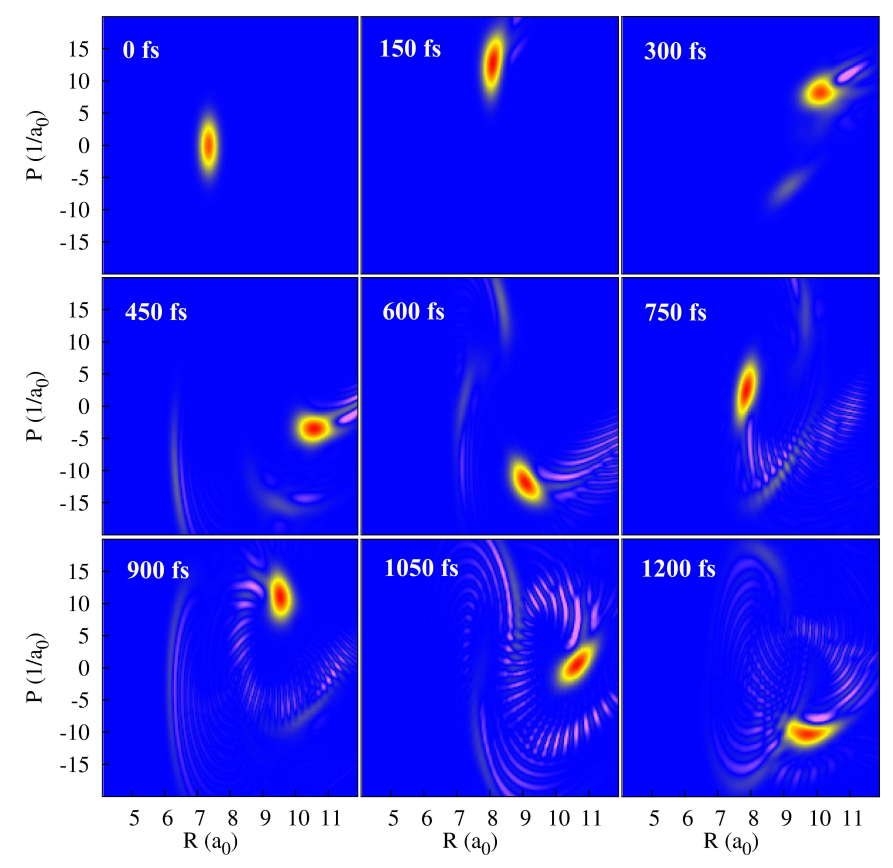

Fig. 7 Quantum mechanical Wigner distribution function for selected timesteps of the dynamics driven by the optimized double pulse from Fig. 5. Violet areas correspond to negative values of the distribution, blue to zero, yellow and red to increasing positive values. 


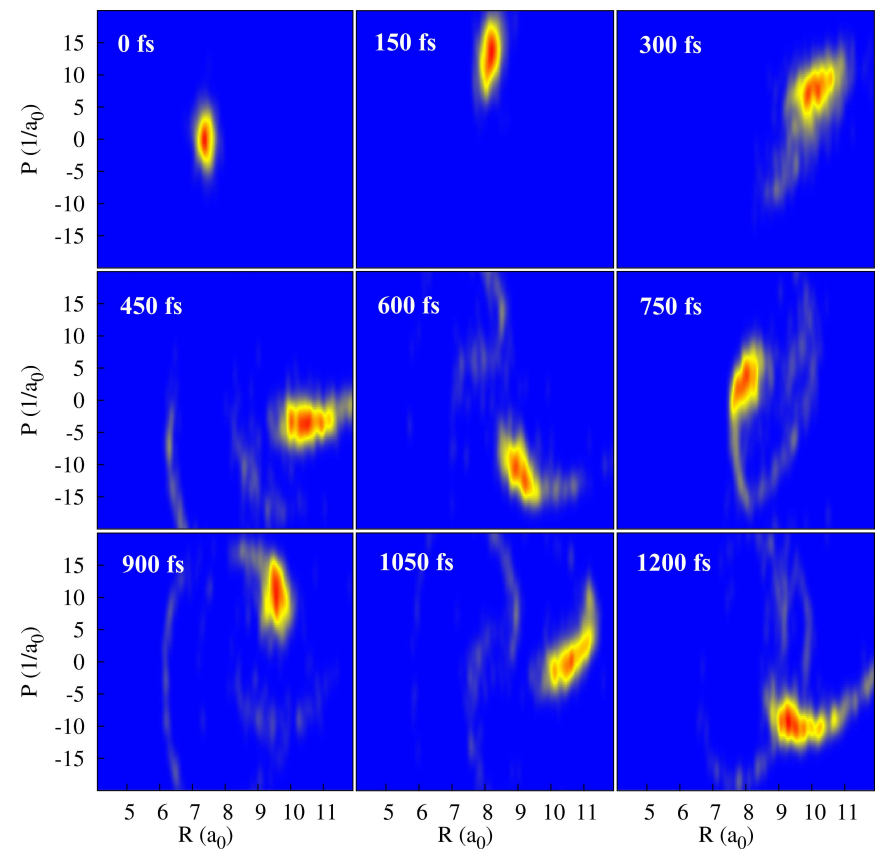

Fig. 8 Classical phase space distribution obtained from FISH simulations for selected timesteps of the dynamics driven by the optimized double pulse from Fig. 5. Blue areas correspond to a value of zero for the distribution, yellow and red to increasing positive values.

The semiclassical FISH phase space densities shown Fig. 8 are obtained by folding the individual trajectories with Gaussian phase space functions of width $0.15 a_{0}$ for the position and $2.5 a_{0}^{-1}$ for the momentum variable. They exhibit very similar evolution as the full quantum mechanical Wigner functions. In particular, the maxima of the phase space density evolve almost identically in the quantum and FISH simulations, whereas the width of the classical distribution becomes slightly larger for later times of the dynamics. Regarding the negative features of the quantum mechanical Wigner function, those belonging to wavepacket motion in less populated electronic states appear also in the semiclassical distribution, but with a positive sign (see e.g. Fig. 8 in the snapshot at 1050 fs the narrow feature at $\mathrm{R} \sim 8.5 a_{0}$ and $\mathrm{P}$ between 5 and 15 $a_{0}^{-1}$ ), whereas those parts corresponding to interferences are not present (e.g. for 1050 fs the periodic feature above the main part of the wavepacket, for R between 9 and $11 a_{0}$ and $\mathrm{P}$ between 4 and $13 a_{0}^{-1}$ ), since they are not described within the semiclassical FISH simulations as discussed above.

\section{Conclusions}

We have demonstrated by comparison with exact quantum dynamics simulations that our recently introduced FISH method can accurately describe coherently driven electron-nuclear dynamics in a system with several coupled electronic states. Moreover, we have shown that the coherent control of a twophoton electronic excitation and selective state populations can be achieved in the framework of the FISH method. This opens the possibility to control photochemistry and photophysics in complex molecular systems since the FISH method has already been implemented in the framework of the ab initio and semiempirical molecular dynamics "on the fly".

\section{Acknowledgement}

We wish to thank Prof. T. Baumert for motivating this work and for fruitful discussions and suggestions. Furthermore we also acknowledge the discussions with Dr. M. Wollenhaupt. This work has been supported by the Deutsche Forschungsgemeinschaft in the framework of the Emmy-Noether Programme, MI-1236 (R. M.) and the Research Unit FOR 1282 (R. M. and J. P.).

\section{References}

1 J. C. Tully, J. Chem. Phys., 1990, 93, 1061.

2 J. C. Tully, Faraday Discuss., 1998, 110, 407.

3 M. Hartmann, J. Pittner and V. Bonačić-Koutecký, J. Chem. Phys., 2001, 114, 2123

4 R. Mitrić, V. Bonačić-Koutecký, J. Pittner and H. Lischka, J. Chem. Phys., 2006, 125, 024303.

5 N. L. Doltsinis and D. Marx, Phys. Rev. Lett., 2002, 88, 166402.

6 G. Granucci, M. Persico and A. Toniolo, J. Chem. Phys., 2001, 114, 10608.

7 U. Werner, R. Mitrić, T. Suzuki and V. Bonačić-Koutecký, Chem. Phys., 2008, 349, 319.

8 R. Mitrić, U. Werner and V. Bonačić-Koutecký, J. Chem. Phys., 2008, 129, 164118 .

9 U. Werner, R. Mitrić and V. Bonačić-Koutecký, J. Chem. Phys., 2010, 132, 174301.

10 T. Fuji, Y.-I. Suzuki, T. Horio, T. Suzuki, R. Mitrić, U. Werner and V. Bonačić-Koutecký, J. Chem. Phys., 2010, 133, 234303.

11 C. F. Craig, W. R. Duncan and O. V. Prezhdo, Phys. Rev. Lett., 2005, 95, 163001.

12 E. Tapavicza, I. Tavernelli and U. Rothlisberger, Phys. Rev. Lett., 2007, 98, 023001.

13 E. Tapavicza, I. Tavernelli, U. Rothlisberger, C. Filippi and M. E. Casida, J. Chem. Phys., 2008, 129, 124108.

14 I. Tavernelli, E. Tapavicza and U. Rothlisberger, J. Chem. Phys., 2009, 130, 124107.

15 E. Fabiano and W. Thiel, J. Phys. Chem. A, 2008, 112, 6859.

16 E. Fabiano, T. W. Keal and W. Thiel, Chem. Phys., 2008, 349, 334.

17 Z. Lan, E. Fabiano and W. Thiel, J. Phys. Chem. B, 2009, 113, 3548.

18 A. Toniolo, S. Olsen, L. Manohar and T. J. Martinez, Farad. Discuss., 2004, 127, 149.

19 A. M. Virshup, C. Punwong, T. V. Pogorelov, B. A. Lindquist, C. Ko and T. J. Martinez, J. Phys. Chem. B, 2009, 113, 3280.

20 L. V. Schäfer, G. Groenhof, M. Boggio-Pasqua, M. A. Robb and H. Grubmüller, PLoS Comp. Biol., 2008, 4, e1000034.

21 G. Groenhof, L. V. Schäfer, M. Boggio-Pasqua, H. Grubmüller and M. A. Robb, J. Am. Chem. Soc., 2008, 130, 3250. 
22 M. Boggio-Pasqua, M. A. Robb and G. Groenhof, J. Am. Chem. Soc., 2009, 131, 13580.

23 R. Mitrić, J. Petersen and V. Bonačić-Koutecký, Phys. Rev. A, 2009, 79, 053416.

24 R. Mitrić, J. Petersen, M. Wohlgemuth, U. Werner, V. Bonačić-Koutecký, L. Wöste and J. Jortner, J. Phys. Chem. A, 2011, 115, 3755.

25 R. Mitrić, J. Petersen, M. Wohlgemuth, U. Werner and V. BonačićKoutecký, Phys. Chem. Chem. Phys., 2011, 13, 8690.

26 J. Stanzel, M. Neeb, W. Eberhardt, P. G. Lisinetskaya, J. Petersen and R. Mitrić, Phys. Rev. A, 2012, 85, 013201.

27 P. G. Lisinetskaya and R. Mitrić, Phys. Rev. A, 2011, 83, 033408.

28 D. J. Tannor and S. A. Rice, J. Chem. Phys., 1985, 83, 5013.

29 D. J. Tannor and S. A. Rice, Adv. Chem. Phys., 1988, 70, 441.

30 P. Brumer and M. Shapiro, Faraday Discuss. Chem. Soc., 1986, 82, 177.

31 M. Shapiro and P. Brumer, J. Chem. Phys., 1986, 84, 4103.

32 M. Shapiro and P. Brumer, Int. Rev. Phys. Chem., 1994, 13, 187.

33 T. Baumert, J. Helbing and G. Gerber, Advances in Chemical Physics, 1997, 101, 47.

34 S. A. Rice and M. Zhao, Optical Control of Molecular Dynamics, John Wiley\& Sons, Inc., New York, 2000.

35 P. W. Brumer and M. Shapiro, Principles of the Quantum Control of Molecular Processes, Wiley-VCH, Berlin, 2003.

36 M. Dantus and V. V. Lozovoy, Chem. Rev., 2004, 104, 1813.

37 Analysis and Control of Ultrafast Photoinduced Reactions, ed. L. Wöste and O. Kühn, Springer Series in Chemical Physics 87, 2007.

38 A. Baltuska, T. Udem, M. Uiberacker, M. Hentschel, E. Goulielmakis, C. Gohle, R. Holzwarth, V. S. Yakovlev, A. Scrinzi, T. W. Hänsch and F. Krausz, Nature, 2003, 421, 611.

39 E. Goulielmakis, V. S. Yakovlev, A. L. Cavalieri, M. Uiberacker, V. Pervak, A. Apolonski, R. Kienberger, U. Kleineberg and F. Krausz, Science, 2007, 317, 769.

40 C. Winterfeldt, C. Spielmann and G. Gerber, Rev. Mod. Phys., 2008, 80, 117.

41 M. Wollenhaupt and T. Baumert, Faraday Discuss., 2011, 153, 9.

42 T. Brixner and G. Gerber, ChemPhysChem, 2003, 4, 418.

43 W. Wohlleben, T. Buckup, J. L. Herek and M. Motzkus, ChemPhysChem, $2005,6,850$.

44 P. Nuernberger, D. Wolpert, H. Weiss and G. Gerber, Proc. Nat. Acad. Sc. USA, 2010, 107, 10366.

45 R. S. Judson and H. Rabitz, Phys. Rev. Lett., 1992, 62, 1500.

46 T. Baumert, T. Brixner, V. Seyfried, M. Strehle and G. Gerber, Appl. Phys. $B, 1997,65,779$.

47 D. Meshulach, D. Yelin and Y. Silberberg, Opt. Commun., 1997, 138, 345.

48 C. J. Bardeen, V. V. Yakovlev, K. R. Wilson, S. D. Carpenter, P. M. Weber and W. S. Warren, Chem. Phys. Lett., 1997, 280, 151.

49 A. Assion, T. Baumert, M. Bergt, T. Brixner, B. Kiefer, V. Seyfried, M. Strehle and G. Gerber, Science, 1998, 282, 919.

50 C. Daniel, J. Full, L. Gonzalez, C. Lupulescu, J. Manz, A. Merli, S. Vajda and L. Wöste, Science, 2003, 299, 536.

51 J. Petersen, R. Mitrić, V. Bonačić-Koutecký, J.-P. Wolf, J. Roslund and H. Rabitz, Phys. Rev. Lett., 2010, 105, 073003.

52 J. Petersen, M. Wohlgemuth, B. Sellner, V. Bonačić-Koutecký, H. Lischka and R. Mitrić, Phys. Chem. Chem. Phys., 2012, DOI:10.1039/C2CP24002E.

53 M. Wollenhaupt, A. Präkelt, C. Sarpe-Tudoran, D. Liese and T. Baumert, J. Opt. B: Quantum Semiclass. Opt., 2005, 7, S270.

54 M. Wollenhaupt, D. Liese, A. Präkelt, C. Sarpe-Tudoran and T. Baumert, Chem. Phys. Lett., 2006, 419, 184.

55 T. Bayer, M. Wollenhaupt, C. Sarpe-Tudoran and T. Baumert, Phys. Rev. Lett., 2009, 102, 023004.

56 M. Wollenhaupt and T. Baumert, J. Photochem. Photobiol. A: Chem., 2006, 180, 248
57 R. Mitrić, J. Petersen and V. Bonačić-Koutecký, Chapter in: Conical Intersections - Theory, Computation and Experiment, W. Domcke, D. R. Yarkony, H. Köppel (Eds.), World Scientific, Singapore, 2011, vol. 17.

58 M. Richter, P. Marquetand, J. Gonzalez-Vazquez, I. Sola and L. Gonzalez, J. Chem. Theory Comput., 2011, 7, 1253.

59 M. Richter, P. Marquetand, J. Gonzalez-Vazquez, I. Sola and L. Gonzalez, J. Chem. Theory Comput., 2012, 8, 374.

60 H.-J. Werner and P. J. Knowles, J. Chem. Phys., 1988, 89, 5803.

61 P. J. Knowles and H.-J. Werner, Chem. Phys. Lett., 1988, 145, 514.

62 P. J. Knowles and H.-J. Werner, Theor. Chim. Acta, 1992, 84, 95.

63 H.-J. Werner, P. J. Knowles, G. Knizia, F. R. Manby, M. Schütz et al., MOLPRO, version 2010.1, a package of ab initio programs, 2010, see http://www.molpro.net.

64 H.-J. Werner and P. J. Knowles, J. Chem. Phys., 1985, 82, 5053.

65 P. J. Knowles and H.-J. Werner, Chem. Phys. Lett., 1985, 115, 259.

66 J. C. Barthelat and P. Durand, Theor. Chim. Acta, 1975, 38, 283.

67 S. Magnier and P. Millié, Phys. Rev. A, 1996, 54, 204.

68 W. Müller and W. Meyer, J. Chem. Phys., 1984, 80, 3311.

69 S. Magnier, M. Aubert-Frécon and A. R. Allouche, J. Chem. Phys., 2004, 121, 1771.

70 R. Kosloff, J. Phys. Chem., 1988, 92, 2087.

71 J. Köhler, M. Wollenhaupt, T. Bayer, C. Sarpe and T. Baumert, Opt. Express, 2011, 19, 11638.

72 L. Verlet, Phys. Rev., 1967, 159, 98.

73 W. C. Swope, H. C. Andersen, P. H. Berens and K. R. Wilson, J. Chem. Phys., 1982, 76, 637.

74 D. E. Goldberg, Genetic Algorithms in Search, Optimization, and Machine Learning, Addison-Wesley, Reading, 1993.

75 E. Wigner, Phys. Rev., 1932, 40, 749. 